\title{
Parametric and nonparametric $A$-Laplace problems: Existence of solutions and asymptotic analysis
}

\author{
Calogero Vetro \\ University of Palermo, Department of Mathematics and Computer Science, Via Archirafi 34, 90123 - \\ Palermo, Italy \\ E-mail: calogero.vetro@unipa.it
}

\begin{abstract}
We give sufficient conditions for the existence of weak solutions to quasilinear elliptic Dirichlet problem driven by the $A$-Laplace operator in a bounded domain $\Omega$. The techniques, based on a variant of the symmetric mountain pass theorem, exploit variational methods. We also provide information about the asymptotic behavior of the solutions as a suitable parameter goes to $0^{+}$. In this case, we point out the existence of a blow-up phenomenon. The analysis developed in this paper extends and complements various qualitative and asymptotic properties for some cases described by homogeneous differential operators.
\end{abstract}

Keywords: Dirichlet boundary value problem, A-Laplace operator, asymptotic analysis, Orlicz-Sobolev space

\section{Introduction}

Let $\Omega \subset \mathbb{R}^{N}$ be a bounded domain with a smooth boundary $\partial \Omega$. In this paper, we study the following quasilinear elliptic Dirichlet problem

$$
-\operatorname{div}(a(|\nabla u|) \nabla u)=f(z, u) \quad \text { in } \Omega, \quad u=0 \quad \text { on } \partial \Omega .
$$

This problem is driven by a differential operator $a(t) t \in C(\mathbb{R})$, the so-called $A$-Laplace operator. We ask about the existence of the solutions in $L^{\infty}(\Omega)$. To this goal the reaction $f(z, t) \in C(\bar{\Omega} \times \mathbb{R})$ obeys assumption $\left(f_{1}\right)$. On the other hand, we impose that $a:[0,+\infty) \rightarrow[0,+\infty)$ satisfy suitable hypotheses to include relevant classes of functions. Motivations arise from the literature review as follows. We recall the nice work of Cencelj-Rădulescu-Repovš [5] on double phase problems in variable exponent Lebesgue-Sobolev spaces, where the authors point out as the study of nonlinear problems is strongly related to the description of significant phenomena in applied sciences (see also PapageorgiouRădulescu-Repovš [12,13,15], and the book of Breit [4, Chapter 2]). For anisotropic double-phase problems we refer to Bahrouni-Rădulescu-Repovš [3], Ragusa-Tachikawa [18], and Zhang-Rădulescu [23].

Here, we mention that existence and multiplicity results for quasilinear elliptic problems were established by Tan-Fang [20], in the Orlicz-Sobolev spaces. Papageorgiou-Vetro [16] proved multiplicity results in variable exponent Lebesgue-Sobolev spaces, Vetro [21] studied semilinear Robin problems of Laplace operator using Lyapunov-Schmidt reduction method, and Vetro [22] considered mixed 
Dirichlet-Neumann problems with the $(p, q)$-Laplace operator. Also, the existence of multiple positive solutions for quasilinear elliptic problems with nonhomogeneous principal part $a$ was established by Fukagai-Narukawa [8] in the Orlicz-Sobolev spaces. Very recently, Alves-De Holanda-Santos [2] proved the existence of positive weak solutions for a semipositone problem driven by a $A$-Laplace operator, with subcritical growth of the reaction.

We recall that the Orlicz spaces are a genuine extension of $L^{p}$ spaces $(1 \leqslant p<+\infty)$, whenever a $N$-function (that is, a convex, even function $A: \mathbb{R} \rightarrow[0,+\infty$ ) satisfying $A(t)=0$ if and only if $t=0, \lim _{t \rightarrow 0} \frac{A(t)}{t}=0$, and $\left.\lim _{t \rightarrow+\infty} \frac{A(t)}{t}=+\infty\right)$ replaces the function $t \rightarrow|t|^{p}$ in the definition of the $L^{p}$ space. Under suitable conditions, Orlicz-Sobolev spaces (extension of the $W^{1, p}$ spaces) are an interesting source of solutions of constrained optimization problems for the energy functional related to (1). Indeed, as stated in Fukagay-Ito-Narukawa [7] the usual Sobolev space is not useful to deal with general forms of the operator $a$ in (1). For example let $a(t) t \in C(\mathbb{R})$ be a function whose primitive is the function $A(t)=\left[1+t^{2}\right]^{\eta}-1$ with $\eta \in \mathbb{R} \backslash\{1\}$, which means nonlinear elasticity in a physical setting if $\eta>1 / 2$. We know that $A(t)$ acts as $2 \eta t^{2}$ as $t$ goes to zero, and acts as $t^{2 \eta}$ as $t$ goes to $\pm \infty$. Thus, the Eulero energy functional associated to problem (1) (namely (6) of Proposition 1 below) cannot be well-defined in both the Sobolev spaces $W_{0}^{1,2}(\Omega)$ and $W_{0}^{1,2 \eta}(\Omega)$ (since no one of these spaces includes the other). This fact motivates the use of the Orlicz-Sobolev space defined in Section 2 to deal with problem (1) (see again [7]).

In this paper we establish some existence results using variational tools together with growth conditions on the reaction. In details the paper is organized as follows. In Section 2 we collect the basic facts on the working spaces and $N$-functions. In Section 3, by using the Palais-Smale condition and a mountain pass theorem for the energy functional associated to problem (1), we establish the existence of at least one nontrivial weak solution of (1) in $C_{0}^{1, \alpha}(\bar{\Omega})$ for some $\alpha \in(0,1)$. The working conditions on the reaction $f$ concern its behavior near zero and at infinity, plus some technical hypotheses. In Section 4 , introducing a parameter in the reaction, we prove two results concerning the asymptotic behavior of the solutions as the parameter goes to zero. For closely related results work, we refer to PapageorgiouVetro-Vetro [17]. Some of the abstract methods used in this paper can be found in the recent monograph Papageorgiou-Rădulescu-Repovš [14].

\section{Mathematical background}

We introduce the function space framework for problem (1). So, we recall some facts on Orlicz and Orlicz-Sobolev spaces (see also Adams-Fournier [1] and Rao-Ren [19]).

For a $N$-function $A: \mathbb{R} \rightarrow[0,+\infty)$, we have the representation

$$
A(t)=\int_{0}^{|t|} \zeta(\xi) d \xi, \quad t \in \mathbb{R}
$$

with $\zeta:[0,+\infty) \rightarrow[0,+\infty)$ being a right derivative of $A$. Also, it is non-decreasing and right continuous such that

$$
\zeta(\xi)>0 \quad \text { for all } \xi>0, \quad \lim _{\xi \rightarrow+\infty} \zeta(\xi)=+\infty, \quad \zeta(0)=0 .
$$


Of course, whenever $\zeta$ meets the above conditions, then $A$, given by (2), is a $N$-function. For our further use, we put $\zeta(\xi)=a(\xi) \xi$ for all $\xi \in[0,+\infty)$ so that (2) reduces to

$$
A(t)=\int_{0}^{|t|} a(\xi) \xi d \xi, \quad t \in \mathbb{R} .
$$

The hypotheses on $a:[0,+\infty) \rightarrow[0,+\infty)$ are as follows:

$\left(a_{1}\right) a \in C^{1}(0,+\infty), a(t)>0,(a(t) t)^{\prime}>0$ for any $t>0$;

$\left(a_{2}\right)$ there exist $q, p \in(1, N)$, with $q \leqslant p<q^{*}$, such that $q \leqslant \frac{A^{\prime}(t) t}{A(t)} \leqslant p$ for any $t>0$, where $A$ is defined by (3) and $q^{*}=N q /(N-q)$;

$\left(a_{3}\right)$ there exist $a_{0}, a_{1}>0$ such that $a_{0} \leqslant \frac{A^{\prime \prime}(t) t}{A^{\prime}(t)} \leqslant a_{1}$ for any $t>0$.

The real function $a(t)=q c t^{q-1}+p C t^{p-1}$ for all $t \in[0,+\infty)$, with $q<p$ and $c, C \geqslant 0$ where $c+C>0$, satisfies the above hypotheses.

We mention that $\left(a_{1}\right)$ and $\left(a_{2}\right)$ imply that $A$ in (3) is a $N$-function which satisfies the inequality

$$
A(2 t) \leqslant k A(t), \quad \text { for all } t>0, \text { some } k>0 \text { (say } \Delta_{2} \text {-condition). }
$$

Now, $A$ admits a conjugate $\widetilde{A}$ given as

$$
\widetilde{A}(t)=\sup \{\tau t-A(\tau): \tau \geqslant 0\} .
$$

Remark 1. Hypothesis $\left(a_{1}\right)$ implies that $\frac{A(s)}{s}$ is increasing for $s>0$ and so

$$
\widetilde{A}\left(\frac{A(s)}{s}\right) \leqslant \frac{A(s)}{s} s=A(s) \text { for } s>0 .
$$

A finite-valued $N$-function $\Psi$ is said to increase essentially more slowly than another $N$-function $A$ near infinity if

$$
\lim _{t \rightarrow+\infty} \frac{\Psi(\lambda t)}{A(t)}=0 \quad \text { for every } \lambda \in \mathbb{R} \text { with } \lambda>0 .
$$

The Orlicz space $L^{A}(\Omega)$, associated with a $N$-function $A$ satisfying the $\Delta_{2}$-condition, is the Banach function space of those measurable functions $u: \Omega \rightarrow \mathbb{R}$ such that the Luxemburg norm

$$
\|u\|_{A}=\inf \left\{\lambda>0: \int_{\Omega} A\left(\frac{|u|}{\lambda}\right) d z \leqslant 1\right\}
$$

is finite. We note that $L^{A}(\Omega)=L^{p}(\Omega)$ if $A(t)=|t|^{p}$ for some $p \in[1,+\infty)$, and $L^{A}(\Omega)=L^{\infty}(\Omega)$ if $A(t)=0$ for $t \in[0,1]$ and $A(t)=+\infty$ for $t>1$. Later on, we denote with $\|\cdot\|_{p}$ the norm in $L^{p}(\Omega)$.

The $\Delta_{2}$-condition leads us to say that the dual space $L^{A}(\Omega)^{*}$ is identified with $L^{\widetilde{A}}(\Omega)$.

We also recall the Hölder type inequality

$$
\int_{\Omega}|u v| d z \leqslant 2\|u\|_{A}\|v\|_{\widetilde{A}} \quad \text { for all } u \in L^{A}(\Omega), \text { all } v \in L^{\widetilde{A}}(\Omega) .
$$


Let $V^{1, A}(\Omega)$ be the Sobolev type space

$$
V^{1, A}(\Omega)=\left\{u: u \text { is weakly differentiable on } \Omega \text { and }|\nabla u| \in L^{A}(\Omega)\right\} .
$$

We consider the Orlicz-Sobolev space $W^{1, A}(\Omega)$ defined by

$$
W^{1, A}(\Omega)=V^{1, A}(\Omega) \cap L^{A}(\Omega),
$$

equipped with the norm $\|u\|_{1, A}=\|\mid \nabla u\|_{A}+\|u\|_{A}$.

As usual, $W_{0}^{1, A}(\Omega)$ stands for the closure in $W^{1, A}(\Omega)$ of the set of smooth compactly supported functions on $\Omega$. Hypothesis $\left(a_{2}\right)$ implies that $\tilde{A}$ satisfies the $\Delta_{2}$-condition. So, $L^{A}(\Omega), W^{1, A}(\Omega)$ and $W_{0}^{1, A}(\Omega)$ are separable and reflexive Banach spaces and the functional

$$
I(u)=\int_{\Omega} A(|\nabla u|) d z \quad \text { for all } u \in W_{0}^{1, A}(\Omega)
$$

is Fréchet differentiable. Hypotheses $\left(a_{1}\right)-\left(a_{3}\right)$ ensure the validity of some elementary inequalities listed in the following lemmas (see $[7,8]$ ).

Lemma 1. If $\left(a_{1}\right),\left(a_{2}\right)$ hold, then whenever $m_{1}(t)=\min \left\{t^{q}, t^{p}\right\}$ and $m_{2}(t)=\max \left\{t^{q}, t^{p}\right\}, t>0$, we have:

(i) $m_{1}(k) A(t) \leqslant A(k t) \leqslant m_{2}(k) A(t)$ for all $k, t \geqslant 0$;

(ii) $m_{1}\left(\|u\|_{A}\right) \leqslant \int_{\Omega} A(|u|) d z \leqslant m_{2}\left(\|u\|_{A}\right)$ for all $u \in L^{A}(\Omega)$.

Lemma 2. If $\left(a_{1}\right)-\left(a_{3}\right)$ hold, one can find $k_{0}>0$ satisfying

$$
(a(|w|) w-a(|v|) v)(w-v) \geqslant k_{0} \frac{A(|w-v|)^{(q+1) / q}}{(A(|w|)+A(|v|))^{1 / q}}
$$

for all $v, w \in \mathbb{R}^{N}$ with $w \neq 0$.

Now, the Poincaré inequality for $A$ can be stated as follows (see the details in Gossez [9], Lemma 2):

There exists $\Theta>0$ such that

$$
\int_{\Omega} A(|u|) d z \leqslant \Theta \int_{\Omega} A(|\nabla u|) d z \quad \text { for all } u \in W_{0}^{1, A}(\Omega) .
$$

We will use $\|\cdot\|=\||\nabla u|\|_{A}$ as the norm of $W_{0}^{1, A}(\Omega)$ (recall that this norm is equivalent to $\|u\|_{1, A}$ ).

By $A_{*}$ we mean the Sobolev's conjugate $N$-function of $A$ given as

$$
A_{*}^{-1}(t)=\int_{0}^{t} \frac{A^{-1}(s)}{s^{(N+1) / N}} d s \quad \text { for } t>0 .
$$

Hypotheses $\left(a_{1}\right)$ and $\left(a_{2}\right)$ imply that $A_{*}$ and $\widetilde{A}_{*}$ are $N$-functions satisfying the $\Delta_{2}$-condition (see [7], Lemma 2.7). Note that

$$
q^{*} \leqslant \frac{A_{*}^{\prime}(t) t}{A_{*}(t)} \leqslant p^{*} \quad \text { for all } t>0 .
$$


We recall that Donaldson-Trudinger [6] showed that there exists a constant $S_{N}>0$ such that

$$
\|u\|_{A_{*}} \leqslant S_{N}\|u\| \quad \text { for all } u \in W_{0}^{1, A}(\Omega),
$$

which means that the embedding $W_{0}^{1, A}(\Omega) \hookrightarrow L^{A_{*}}(\Omega)$ is continuous. If $\Omega$ is a bounded domain and $B$ is a $N$-function satisfying

$$
\limsup _{t \rightarrow+\infty} \frac{B(t)}{A_{*}(t)}=0
$$

then $W_{0}^{1, A}(\Omega) \hookrightarrow L^{B}(\Omega)$ is a compact embedding. In particular, $W_{0}^{1, A}(\Omega) \hookrightarrow L^{A}(\Omega)$ is compact too.

Remark 2. We note that Lemma 1(ii) implies:

(j) $\int_{\Omega} A(|u|) d z<+\infty$ for all $u \in L^{A}(\Omega)$;

(jj) a sequence $\left\{u_{n}\right\}_{n \geqslant 1} \subset L^{A}(\Omega)$ converges to some $u \in L^{A}(\Omega)$ if and only if

$$
\lim _{n \rightarrow+\infty} \int_{\Omega} A\left(\left|u_{n}-u\right|\right) d z=0
$$

(jjj) a sequence $\left\{u_{n}\right\}_{n} \geqslant 1 \subset L^{A}(\Omega)$ is bounded in $L^{A}(\Omega)$ if and only if

$$
\left\{\int_{\Omega} A\left(\left|u_{n}\right|\right) d z\right\}_{n \geqslant 1} \text { is bounded. }
$$

Moreover, we point out that $A$ increases essentially more slowly than $A_{*}$ near infinity. In fact, for $t \geqslant 1$ we have

$$
0 \leqslant \frac{A(\lambda t)}{A_{*}(t)} \leqslant \frac{A(\lambda) t^{p}}{A_{*}(1) t^{*}} \rightarrow 0 \text { as } t \rightarrow+\infty, \text { since } p<q^{*} .
$$

The next lemma states a convergence result in a Orlicz-Sobolev space.

Lemma 3. Let $\Omega \subset \mathbb{R}^{N}$ be a smooth bounded domain, and suppose that $\left(a_{1}\right)-\left(a_{2}\right)$ hold true. If $u \in$ $W_{0}^{1, A}(\Omega)$ and $\left\{u_{n}\right\}_{n \geqslant 1}$ is such that $u_{n} \stackrel{w}{\rightarrow} u$ in $W_{0}^{1, A}(\Omega)$ and

$$
\lim _{n \rightarrow+\infty} \int_{\Omega} a\left(\left|\nabla u_{n}\right|\right) \nabla u_{n}\left(\nabla u_{n}-\nabla u\right) d z=0
$$

then $u_{n}$ converges to $u$ in $W_{0}^{1, A}(\Omega)$.

Proof. Firstly, we note that $u_{n} \stackrel{w}{\rightarrow} u$ in $W_{0}^{1, A}(\Omega)$ yields

$$
\lim _{n \rightarrow+\infty} \int_{\Omega} a(|\nabla u|) \nabla u \nabla\left(u_{n}-u\right) d z=0 .
$$


So, we get

$$
\lim _{n \rightarrow+\infty} \int_{\Omega}\left(a\left(\left|\nabla u_{n}\right|\right) \nabla u_{n}-a(|\nabla u|) \nabla u\right)\left(\nabla u_{n}-\nabla u\right) d z=0 .
$$

Since the sequence $\left\{u_{n}\right\}_{n} \geqslant 1$ is bounded, the Hölder inequality and Lemma 2 lead to

$$
\begin{aligned}
& \int_{\Omega} A\left(\left|\nabla u_{n}-\nabla u\right|\right) d z \\
& \quad \leqslant\left(\int_{\Omega} \frac{A\left(\left|\nabla u_{n}-\nabla u\right|\right)^{(q+1) / q}}{\left(A\left(\left|\nabla u_{n}\right|\right)+A(|\nabla u|)\right)^{1 / q}} d z\right)^{q /(q+1)}\left(\int_{\Omega}\left(A\left(\left|\nabla u_{n}\right|\right)+A(|\nabla u|)\right) d z\right)^{1 /(q+1)} \\
& \quad \leqslant M\left(\frac{1}{k_{0}} \int_{\Omega}\left(a\left(\left|\nabla u_{n}\right|\right) \nabla u_{n}-a(|\nabla u|) \nabla u\right)\left(\nabla u_{n}-\nabla u\right) d z\right)^{q /(q+1)} \quad \text { for some } M>0 \\
& \quad \rightarrow 0 \text { as } n \rightarrow+\infty .
\end{aligned}
$$

So, by Remark 2(jj), we conclude that $u_{n} \rightarrow u$ in $W_{0}^{1, A}(\Omega)$, as $n \rightarrow+\infty$.

\section{One nontrivial weak solution}

We recall that $u \in W_{0}^{1, A}(\Omega)$ is a weak solution of (1) whenever

$$
\int_{\Omega} a(|\nabla u|) \nabla u \nabla v d z=\int_{\Omega} f(z, u) v d z \quad \text { for any } v \in W_{0}^{1, A}(\Omega) .
$$

For the sake of clarity, we recall the Palais-Smale condition too.

Definition 1. Let $W_{0}^{1, A}(\Omega)^{*}$ be the topological dual of $W_{0}^{1, A}(\Omega)$. Then, $I: W_{0}^{1, A}(\Omega) \rightarrow \mathbb{R}$ satisfies the Palais-Smale condition if any sequence $\left\{u_{n}\right\}_{n} \geqslant 1$ such that

(i) $\left\{I\left(u_{n}\right)\right\}_{n} \geqslant 1$ is bounded;

(ii) $\lim _{n \rightarrow+\infty}\left\|I^{\prime}\left(u_{n}\right)\right\|_{W_{0}^{1, A}(\Omega)^{*}}=0$,

has a convergent subsequence.

A sequence $\left\{u_{n}\right\}_{n} \geqslant 1$ satisfying Definition 1(i)-(ii), is called a Palais-Smale sequence for the functional $I$. Here, we use the following inequality:

$$
\limsup _{|t| \rightarrow+\infty}\left(\sup _{z \in \Omega} \frac{\beta F(z, t)-t f(z, t)}{A(|t|)}\right)<\frac{\beta-p}{\Theta}
$$

for some $\beta>p$ where $\Theta$ is as in (4) and $F(z, t)=\int_{0}^{t} f(z, \xi) d \xi$.

Now, we consider the following condition (see assumption $\left(f_{*}\right)$ of [20]): 
$\left(f_{1}\right) f(z, 0)=0$ for all $z \in \bar{\Omega}$ and there are a $N$-function $B$ such that $B^{\prime}: \mathbb{R} \rightarrow \mathbb{R}$ is an odd increasing homeomorphism, and constants $\alpha_{0}, \alpha_{1} \geqslant 0$ with $|f(z, t)| \leqslant \alpha_{0}+\alpha_{1} B^{\prime}(|t|)$ for all $z \in \bar{\Omega}$, $t \in \mathbb{R}$, and

$$
\lim _{t \rightarrow+\infty} \frac{B(t)}{A_{*}(t)}=0
$$

and

$$
p<b^{-}:=\inf _{t>0} \frac{t B^{\prime}(t)}{B(t)} \leqslant \sup _{t>0} \frac{t B^{\prime}(t)}{B(t)}:=b^{+}<q^{*} .
$$

We establish the following result.

Proposition 1. If $\left(f_{0}\right),\left(f_{1}\right)$ hold, then the functional $I: W_{0}^{1, A}(\Omega) \rightarrow \mathbb{R}$ defined by

$$
I(u)=\int_{\Omega} A(|\nabla u|) d z-\int_{\Omega} F(z, u) d z \quad \text { for all } u \in W_{0}^{1, A}(\Omega)
$$

satisfies the Palais-Smale condition.

Proof. Using $\left(f_{0}\right)$, we choose $\rho \in\left(0, \frac{\beta-p}{\Theta}\right)$ such that

$$
\rho>\limsup _{|t| \rightarrow+\infty}\left(\sup _{z \in \Omega} \frac{\beta F(z, t)-t f(z, t)}{A(|t|)}\right) .
$$

Then, we can find $t^{*}>0$ such that

$$
\beta F(z, t)-t f(z, t) \leqslant \rho A(|t|) \text { for all }|t| \geqslant t^{*}, z \in \Omega .
$$

So, there exists $\delta>0$ satisfying

$$
\beta F(z, t)-t f(z, t) \leqslant \rho A(|t|)+\delta \quad \text { for all } t \in \mathbb{R}, z \in \Omega .
$$

Let $\left\{u_{n}\right\}_{n \geqslant 1}$ be a Palais-Smale sequence in $W_{0}^{1, A}(\Omega)$ for the functional $I$. Set $\varepsilon_{n}:=\left\|I^{\prime}\left(u_{n}\right)\right\|$. Since $\left\{I\left(u_{n}\right)\right\}_{n \geqslant 1}$ is bounded, (6) and

$$
\left\langle I^{\prime}\left(u_{n}\right), v\right\rangle=\int_{\Omega} a\left(\left|\nabla u_{n}\right|\right) \nabla u_{n} \nabla v d z-\int_{\Omega} f\left(z, u_{n}\right) v d z \quad \text { for all } u_{n}, v \in W_{0}^{1, A}(\Omega), n \in \mathbb{N},
$$

imply that we can find a constant $L$ satisfying

$$
\begin{aligned}
L+\varepsilon_{n}\left\|u_{n}\right\| & =L+\varepsilon_{n}\left\|\left|\nabla u_{n}\right|\right\|_{A} \\
& \geqslant \beta I\left(u_{n}\right)-\left\langle I^{\prime}\left(u_{n}\right), u_{n}\right\rangle \\
& =\beta \int_{\Omega} A\left(\left|\nabla u_{n}\right|\right) d z-\beta \int_{\Omega} F\left(z, u_{n}\right) d z-\int_{\Omega} a\left(\left|\nabla u_{n}\right|\right)\left|\nabla u_{n}\right|^{2} d z+\int_{\Omega} f\left(z, u_{n}\right) u_{n} d z
\end{aligned}
$$




$$
\begin{aligned}
& \left.\geqslant(\beta-p) \int_{\Omega} A\left(\left|\nabla u_{n}\right|\right) d z-\int_{\Omega}\left[\beta F\left(z, u_{n}\right)-f\left(z, u_{n}\right) u_{n}\right] d z \quad \text { (by }\left(a_{2}\right)\right) \\
& \geqslant(\beta-p) \int_{\Omega} A\left(\left|\nabla u_{n}\right|\right) d z-\rho \int_{\Omega} A\left(\left|u_{n}\right|\right) d z-\delta|\Omega| \quad(\text { by }(7)) \\
& \geqslant(\beta-p) \int_{\Omega} A\left(\left|\nabla u_{n}\right|\right) d z-\rho \Theta \int_{\Omega} A\left(\left|\nabla u_{n}\right|\right) d z-\delta|\Omega| \quad(\text { by }(4)) \\
& \geqslant(\beta-p-\rho \Theta) \int_{\Omega} A\left(\left|\nabla u_{n}\right|\right) d z-\delta|\Omega| \\
& \geqslant(\beta-p-\rho \Theta) m_{1}\left(\left\|\left|\nabla u_{n}\right|\right\|_{A}\right)-\delta|\Omega| \\
& \geqslant(\beta-p-\rho \Theta)\left\|\left|\nabla u_{n}\right|\right\|_{A}^{q}-\delta|\Omega| \quad\left(\text { by Lemma 1, if }\left\|\left|\nabla u_{n}\right|\right\|_{A} \geqslant 1\right)
\end{aligned}
$$

where $|\Omega|$ is the Lebesgue measure of $\Omega$. If the sequence $\left\{\left\|u_{n}\right\|\right\}_{n} \geqslant 1$ is not bounded, from

$$
L+\varepsilon_{n}\left\|u_{n}\right\|=L+\varepsilon_{n}\left\|\left|\nabla u_{n}\right|\right\|_{A} \geqslant(\alpha-p-\rho \Theta)\left\|\left|\nabla u_{n}\right|\right\|_{A}^{q}-\delta|\Omega|
$$

for infinite values of $n$ large enough,

we obtain a contradiction (recall that $\left\|u_{n}\right\|=\left\|\left|\nabla u_{n}\right|\right\|_{A}$ ). So, the sequence $\left\{u_{n}\right\}_{n} \geqslant 1$ is bounded in $W_{0}^{1, A}(\Omega)$. Consequently, $\left\{u_{n}\right\}_{n} \geqslant 1$ admits a subsequence (namely $\left\{u_{n}\right\}_{n} \geqslant 1$ too) such that

$$
u_{n} \stackrel{w}{\rightarrow} u \text { in } W_{0}^{1, A}(\Omega) \text { and } u_{n} \rightarrow u \text { in } L^{B}(\Omega) \quad\left(\text { recall }(5) \text {, since } B(t) / A_{*}(t) \rightarrow 0 \text { as } t \rightarrow+\infty\right) .
$$

We note that the condition $\left(f_{1}\right)$ ensures:

- $f\left(\cdot, u_{n}(\cdot)\right) \in L^{\widetilde{B}}(\Omega)$ for all $n \in \mathbb{N}$, where $\widetilde{B}$ is the conjugate of $B$;

- $\left\{f\left(\cdot, u_{n}(\cdot)\right)\right\}_{n \geqslant 1}$ is bounded in $L^{\widetilde{B}}(\Omega)$.

Using Hölder inequality, we infer that

$$
\lim _{n \rightarrow+\infty} \int_{\Omega}\left|f\left(z, u_{n}\right)\right|\left|u_{n}-u\right| d z=0 .
$$

From

$$
\int_{\Omega} a\left(\left|\nabla u_{n}\right|\right) \nabla u_{n} \nabla\left(u_{n}-u\right) d z=\left\langle I^{\prime}\left(u_{n}\right), u_{n}-u\right\rangle+\int_{\Omega} f\left(z, u_{n}\right)\left(u_{n}-u\right) d z,
$$

we get

$$
\lim _{n \rightarrow+\infty} \int_{\Omega} a\left(\left|\nabla u_{n}\right|\right) \nabla u_{n} \nabla\left(u_{n}-u\right) d z=0
$$

and by Lemma 3, we conclude that $u_{n} \rightarrow u$ in $W_{0}^{1, A}(\Omega)$ as $n \rightarrow+\infty$.

Remark 3. Note that the condition $\left(f_{0}\right)$ is motivated by Assumption 2.1(iv) of [10]. Also, $\left(f_{0}\right)$ is weaker than the Ambrosetti-Rabinowitz condition (see Remark 2.3 of [10]). 
In the sequel we will use the following conditions:

$\left(f_{2}\right)$ there exist $\varepsilon \in\left(0, \Theta^{-1}\right)$ and $\delta_{\varepsilon}>0$ such that $F(z, t) \leqslant \varepsilon A(|t|)$ for a.a. $z \in \Omega$, all $|t| \leqslant \delta_{\varepsilon}$;

$\left(f_{3}\right) \lim _{|t| \rightarrow+\infty} \frac{F(z, t)}{|t|^{p}}=+\infty$ uniformly for a.a. $z \in \Omega$;

$\left(f_{3}^{+}\right) \lim _{t \rightarrow+\infty} \frac{F(z, t)}{t^{p}}=+\infty$ uniformly for a.a. $z \in \Omega$;

$\left(f_{3}^{-}\right) \lim _{t \rightarrow-\infty} \frac{F(z, t)}{|t|^{p}}=+\infty$ uniformly for a.a. $z \in \Omega$.

We establish our next result in the form of a lemma.

Lemma 4. If $\left(f_{1}\right),\left(f_{2}\right)$ hold and $f$ satisfies also $\left(f_{3}^{+}\right)$or $\left(f_{3}^{-}\right)$, then

(i) there exist $\rho>0$ and $\sigma>0$ such that $I(u) \geqslant \sigma$ for each $u \in W_{0}^{1, A}(\Omega)$ with $\|u\|=\rho$;

(ii) there exists $v \in W_{0}^{1, A}(\Omega)$ such that $0>I(v)$ and $\rho<\|v\|$.

Proof. (i). Since $W_{0}^{1, A}(\Omega) \hookrightarrow L^{B}(\Omega)$ continuously, there is a constant $C_{B}>0$ satisfying

$$
\|u\|_{B} \leqslant C_{B}\|u\| \quad \text { for all } u \in W_{0}^{1, A}(\Omega) .
$$

Using $\left(f_{1}\right)$ and $\left(f_{2}\right)$, we can find a constant $C_{\varepsilon}>0$ satisfying

$$
F(z, t) \leqslant \varepsilon A(|t|)+C_{\varepsilon} B(|t|) \text { for a.a. } z \in \Omega \text {, all } t \in \mathbb{R} \text {. }
$$

If $u \in W_{0}^{1, A}(\Omega)$ is such that $\max \left\{\|u\|, C_{B}\|u\|\right\}<1$, by (8) and (9) we have

$$
\begin{aligned}
I(u) & =\int_{\Omega} A(|\nabla u|) d z-\int_{\Omega} F(z, u) d z \\
& \geqslant \int_{\Omega} A(|\nabla u|) d z-\varepsilon \int_{\Omega} A(|u|) d z-C_{\varepsilon} \int_{\Omega} B(|u|) d z \\
& \geqslant(1-\varepsilon \Theta) \int_{\Omega} A(|\nabla u|) d z-C_{\varepsilon}\|u\|_{B}^{b^{-}} \quad(\text { see (4) and Lemma 1(ii)) } \\
& \geqslant(1-\varepsilon \Theta) m_{1}\left(\||\nabla u|\|_{A}\right)-C_{\varepsilon} C_{B}^{b^{-}}\|u\|^{b^{-}} \\
& =(1-\varepsilon \Theta)\|u\|^{p}-C_{\varepsilon} C_{B}^{b^{-}}\|u\|^{b^{-}} \\
& =\left[(1-\varepsilon \Theta)-C_{\varepsilon} C_{B}^{b^{-}}\|u\|^{b^{-}-p}\right]\|u\|^{p} .
\end{aligned}
$$

Choosing $0<\rho<\min \left\{1, C_{B}^{-1}\right\}$ with

$$
\vartheta=(1-\varepsilon \Theta)-C_{\varepsilon} C_{B}^{b^{-}} \rho^{b^{-}-p}>0,
$$

then we have $I(u) \geqslant \vartheta \rho^{p}=\sigma>0$ for all $u \in W_{0}^{1, A}(\Omega)$ such that $\|u\|=\rho$.

(ii). Assume that $f$ satisfies $\left(f_{3}^{+}\right)$. By $\left(f_{1}\right)$ and $\left(f_{3}^{+}\right)$, for all $L>0$ we can find a constant $C_{L}>0$ satisfying

$$
F(z, t) \geqslant L t^{p}-C_{L} \quad \text { for a.a. } z \in \Omega \text {, all } t>0 .
$$


Set $w \in W_{0}^{1, A}(\Omega)$ with $w(z)>0$ for all $z \in \Omega$. From (10), for all $t>1$ we have

$$
\begin{aligned}
I(t w) & =\int_{\Omega} A(|\nabla t w|) d z-\int_{\Omega} F(z, t w) d z \\
& \leqslant t^{p} \int_{\Omega} A(|\nabla w|) d z-L t^{p}\|w\|_{p}^{p}+C_{L}|\Omega| \\
& =t^{p}\left[\int_{\Omega} A(|\nabla w|) d z-L\|w\|_{p}^{p}\right]+C_{L}|\Omega| .
\end{aligned}
$$

Choosing $L>0$ with

$$
\int_{\Omega} A(|\nabla w|) d z-L\|w\|_{p}^{p}<0
$$

then $I(t w) \rightarrow-\infty$ as $t \rightarrow+\infty$. Consequently there is $v=t_{0} w \in W_{0}^{1, A}(\Omega)$ with $0>I(v)$ and $\rho<\|v\|$.

The same conclusion holds if we assume that $f$ satisfies $\left(f_{3}^{-}\right)$.

For reader convenience, we recall the following version of Mountain Pass Theorem (see Theorem 5.40 of [11]).

Theorem 1. If $I \in C^{1}\left(W_{0}^{1, A}(\Omega)\right)$ satisfies the $\left(C_{c}\right)$-condition, there exist $u_{0}, u_{1} \in W_{0}^{1, A}(\Omega)$ and $\rho>0$ such that

$$
\begin{aligned}
& \left\|u_{0}-u_{1}\right\|>\rho, \quad \max \left\{I\left(u_{0}\right), I\left(u_{1}\right)\right\}<\inf \left\{I(u):\left\|u-u_{0}\right\|=\rho\right\}=m_{\rho}, \quad \text { and } \\
& c=\inf _{\gamma \in \Gamma} \max _{0 \leqslant t \leqslant 1} I(\gamma(t)) \quad \text { with } \Gamma=\left\{\gamma \in C\left([0,1], W_{0}^{1, A}(\Omega)\right): \gamma(0)=u_{0}, \gamma(1)=u_{1}\right\},
\end{aligned}
$$

then $c \geqslant m_{\rho}$ and $c$ is a critical value of $I$ (that is, there exists $\widehat{u} \in W_{0}^{1, A}(\Omega)$ such that $I^{\prime}(\widehat{u})=0$ and $I(\widehat{u})=c)$.

Remark 4. We recall that $I \in C^{1}\left(W_{0}^{1, A}(\Omega)\right)$ satisfies the $\left(C_{c}\right)$-condition, if every sequence $\left\{u_{n}\right\}_{n \geqslant 1} \subset$ $W_{0}^{1, A}(\Omega)$ such that $I\left(u_{n}\right) \rightarrow c \in \mathbb{R}$ and $\left(1+\left\|u_{n}\right\|_{A}\right) I^{\prime}\left(u_{n}\right) \rightarrow 0$ in $W_{0}^{1, A}(\Omega)^{*}$ as $n \rightarrow+\infty$, admits a convergent subsequence. Note that if $I$ satisfies the Palais-Smale condition then it satisfies the $\left(C_{c}\right)$ condition.

By Proposition 1, Lemma 4 and Remark 4, the functional $I$ defined in (6) satisfies the assumptions of Theorem 1. So, it admits a critical value $c \geqslant m_{\rho}>0$.

Resuming we establish the existence of one nontrivial weak solution of (1) in the following result. By Corollary 3.1 of [20] this solution is in $C_{0}^{1, \alpha}(\bar{\Omega})$ for some $\alpha \in(0,1)$.

Theorem 2. If $\left(f_{0}\right)-\left(f_{2}\right),\left(f_{3}^{+}\right)$(or $\left.\left(f_{3}^{-}\right)\right)$hold, then problem (1) admits at least one nontrivial weak solution $\widehat{u} \in C_{0}^{1, \alpha}(\bar{\Omega})$ for some $\alpha \in(0,1)$. 


\section{The parametric case: Existence and blow-up of solutions}

In this section, we study the following parametric version of problem (1):

$$
-\operatorname{div}(a(|\nabla u|) \nabla u)=\lambda f(z, u) \quad \text { in } \Omega, \quad u=0 \quad \text { on } \partial \Omega,
$$

where $\lambda>0$. In particular, we are interested in the existence of high energy solutions, that is, solutions with higher and higher energies as the positive parameter becomes smaller and smaller.

As a consequence of Theorem 2 we deduce the following existence result.

Theorem 3. If $\left(f_{0}\right)-\left(f_{2}\right),\left(f_{3}^{+}\right)$(or $\left.\left(f_{3}^{-}\right)\right)$hold, then problem (11) admits for all $\lambda \in(0,1]$ at least one nontrivial weak solution $\widehat{u}_{\lambda} \in C_{0}^{1, \alpha}(\bar{\Omega})$, for some $\alpha \in(0,1)$.

Now, we show that for small values of the parameter $\lambda>0$ problem (1) has a solution $u_{\lambda} \in W_{0}^{1, A}(\Omega)$ such that $\lim _{\lambda \rightarrow 0^{+}}\left\|u_{\lambda}\right\|=+\infty$.

Lemma 5. If $\left(f_{1}\right)$ holds, then there exist positive constants $m_{\lambda}$ and $\rho_{\lambda}$ such that $\lim _{\lambda \rightarrow 0^{+}} m_{\lambda}=+\infty$ and $I_{\lambda}(u) \geqslant m_{\lambda}>0$ for all $u \in W_{0}^{1, A}(\Omega)$ such that $\|u\|=\rho_{\lambda}$.

Proof. Let $u \in W_{0}^{1, A}(\Omega)$ with $\|u\|>1$. From $\left(f_{1}\right)$, we deduce that there is $C>0$ with

$$
|F(z, t)| \leqslant C(1+B(|t|)),
$$

for all $(x, t) \in \Omega \times \mathbb{R}, p<b^{-} \leqslant b^{+}<q^{*}$. Consequently, we have

$$
\begin{aligned}
I_{\lambda}(u) & =\int_{\Omega} A(|\nabla u|) d z-\lambda \int_{\Omega} F(z, u) d z \\
& \geqslant \int_{\Omega} A(|\nabla u|) d z-\lambda C \int_{\Omega} B(|u|) d z-\lambda C|\Omega| \\
& \geqslant m_{1}(\||\nabla u|\|)-\lambda C m_{2}\left(\|u\|_{B}\right)-\lambda C|\Omega| \quad(\text { see Lemma 1(ii)) } \\
& \geqslant\|u\|^{q}-\lambda C m_{2}\left(C_{B}\|u\|\right)-\lambda C|\Omega| \\
& \geqslant\|u\|^{q}-\lambda C \max \left\{C_{B}^{b^{+}}, C_{B}^{b^{-}}\right\}\|u\|^{b^{+}}-\lambda C|\Omega| .
\end{aligned}
$$

Let $\rho_{\lambda}=\lambda^{-\sigma}$ with $0<\sigma<\frac{1}{b^{+}-q}$, so that $\rho_{\lambda}>1$ for $\lambda>0$ small enough. Putting $\|u\|=\rho_{\lambda}=\lambda^{-\sigma}$ in the above inequality, we get

$$
I_{\lambda}(u) \geqslant \lambda^{-\sigma q}-C \max \left\{C_{B}^{b^{+}}, C_{B}^{b^{-}}\right\} \lambda^{1-\sigma b^{+}}-\lambda C|\Omega| .
$$

Now, set $m_{\lambda}=\lambda^{-\sigma q}-C \max \left\{C_{B}^{b^{+}}, C_{B}^{b^{-}}\right\} \lambda^{1-\sigma b^{+}}-\lambda C|\Omega|$. As $0<\sigma<\frac{1}{b^{+}-q}$, then we can find $\lambda_{0}$ small enough such that $m_{\lambda}>0$ for all $0<\lambda<\lambda_{0}$ and $m_{\lambda} \rightarrow+\infty$ as $\lambda \rightarrow 0^{+}$.

Theorem 4. If $\left(f_{0}\right),\left(f_{1}\right),\left(f_{3}\right)$ hold, then there exists $\lambda_{0} \in(0,1]$ such that, for all $0<\lambda<\lambda_{0}$, Problem (11) has at least one weak solution $u_{\lambda} \in W_{0}^{1, A}(\Omega)$ and $\left\|u_{\lambda}\right\| \rightarrow+\infty$ as $\lambda \rightarrow 0^{+}$. 
Proof. By Proposition 1 , the functional $I_{\lambda}$ satisfies the $\left(C_{c}\right)$-condition for all $\lambda \in(0,1]$. Thanks to Proposition 1, Lemma 5 and Lemma 4(ii) all the hypotheses of the mountain pass theorem are satisfied and so, there exists a nontrivial critical point $u_{\lambda}$ for $I_{\lambda}$ such that

$$
I_{\lambda}\left(u_{\lambda}\right)=c_{\lambda} \geqslant m_{\lambda}
$$

On the other hand, from (12), we have

$$
\begin{aligned}
I_{\lambda}\left(u_{\lambda}\right) & \leqslant \int_{\Omega} A\left(\left|\nabla u_{\lambda}\right|\right) d z+\lambda \int_{\Omega}\left|F\left(z, u_{\lambda}\right)\right| d z \\
& \leqslant m_{2}\left(\left\|\nabla u_{\lambda}\right\|_{A}\right)+\lambda C m_{2}\left(\left\|u_{\lambda}\right\|_{B}\right)+\lambda C|\Omega| .
\end{aligned}
$$

Taking the limit as $\lambda \rightarrow 0^{+}$in the previous inequality, and using Lemma 5 one has $\lim _{\lambda \rightarrow 0^{+}}\left\|u_{\lambda}\right\|=$ $+\infty$.

The new condition on the function $f(z, t)$ in the reaction is the following:

$\left(f_{4}\right)$ : There exists $\tau \in(1, p)$ and $\delta, \widehat{c}$ such that

$$
\widehat{c}|t|^{\tau} \leqslant F(z, t) \text { for a.a. } z \in \Omega, \text { all }|t| \leqslant \delta .
$$

Theorem 5. If hypotheses $\left(f_{1}\right),\left(f_{4}\right)$ hold, then we can find $\widehat{\lambda} \in(0,1)$ such that for all $\lambda \in(0, \widehat{\lambda})$ problem (11) has a nontrivial solution $\widehat{u}_{\lambda} \in W_{0}^{1, A}(\Omega)$ and $\left\|\widehat{u}_{\lambda}\right\| \rightarrow 0^{+}$as $\lambda \rightarrow 0^{+}$.

Proof. We consider again the functional $I_{\lambda}: W_{0}^{1, A}(\Omega) \rightarrow \mathbb{R}$ related to problem (11) and given as

$$
I_{\lambda}(u)=\int_{\Omega} A(|\nabla u|) d z-\lambda \int_{\Omega} F(z, u) d z \quad \text { for all } u \in W_{0}^{1, A}(\Omega) .
$$

We know that there is $C_{\tau}>0$ such that $\|u\|_{\tau} \leqslant C_{\tau}\|u\|$. Hypotheses $\left(f_{1}\right),\left(f_{4}\right)$ imply that

$$
|F(z, t)| \leqslant C\left[|t|^{\tau}+B(|t|)\right] \text { for a.a. } z \in \Omega \text {, all } t \in \mathbb{R} \text {, some } C>0 \text {. }
$$

Let $0<\sigma<\frac{1}{p}$. Then for $u \in W_{0}^{1, A}(\Omega)$ with $\|u\|=\lambda^{\sigma}<1$, we have

$$
\begin{aligned}
I_{\lambda}(u) & \geqslant \int_{\Omega} A(|\nabla u|) d z-\lambda C\|u\|_{\tau}^{\tau}-\lambda C \int_{\Omega} B(|u|) d z \quad(\text { see }(13)) \\
& \geqslant m_{1}(\||\nabla u|\|)-\lambda C\|u\|_{\tau}^{\tau}-\lambda C m_{2}\left(C_{B}\|u\|\right) \\
& =\|u\|^{p}-\lambda C C_{\tau}^{\tau}\|u\|^{\tau}-\lambda C \max \left\{C_{B}^{b^{+}}, C_{B}^{b^{-}}\right\}\|u\|^{b^{-}}
\end{aligned}
$$

As $\sigma p-1<0$, then one can find $\widehat{\lambda}>0$ such that for all $\lambda \in(0, \widehat{\lambda})$ we get

$$
I_{\lambda}(u)>0 \quad \text { for all } u \in W_{0}^{1, A}(\Omega) \text { with }\|u\|=\lambda^{\sigma} .
$$


Let $B_{\lambda}=\left\{u \in W_{0}^{1, A}(\Omega):\|u\|<\lambda^{\sigma}\right\}$. The reflexivity of $W_{0}^{1, A}(\Omega)$ and the Eberlein-Smulian theorem imply that $\bar{B}_{\lambda}$ is sequentially weakly compact. Now, $I_{\lambda}$ is sequentially weakly lower semicontinuous (note that $\left.W_{0}^{1, A}(\Omega)\right) \hookrightarrow L^{p}(\Omega)$ compactly). By the Weierstrass-Tonelli theorem, we have $\widehat{u}_{\lambda} \in W_{0}^{1, A}(\Omega)$ such that

$$
I_{\lambda}\left(\widehat{u}_{\lambda}\right)=\min \left[I_{\lambda}(u): u \in \bar{B}_{\lambda}\right]
$$

Let $u \in C_{0}^{1}(\bar{\Omega}) \subset W_{0}^{1, A}(\Omega)$ with $u(z)>0$ for all $z \in \Omega$. Then we can find $t \in(0,1)$ small such that $0<t u(z) \leqslant \delta$ for all $z \in \Omega$, where $\delta>0$ is as postulated by hypothesis $H_{2}(\mathrm{ii})$. We have

$$
\begin{aligned}
I_{\lambda}(t u) & \leqslant \int_{\Omega} A(t|u|) d z-\lambda C\|t u\|_{\tau}^{\tau} \quad\left(\text { see hypothesis } H_{2}(\mathrm{ii})\right) \\
& \leqslant m_{2}(t) \int_{\Omega} A(|u|) d z-\lambda C t^{\tau}\|u\|_{\tau}^{\tau} \\
& =t^{\tau}\left[t^{p-\tau} \int_{\Omega} A(|u|) d z-\lambda C\|u\|_{\tau}^{\tau}\right] .
\end{aligned}
$$

Since $1<\tau<p$, choosing $t \in(0,1)$ even smaller if necessary, we have

$$
\begin{aligned}
& I_{\lambda}(t u)<0, \\
& \quad \Rightarrow \quad I_{\lambda}\left(\widehat{u}_{\lambda}\right)<0=I_{\lambda}(0) \quad(\text { see }(15)), \\
& \quad \Rightarrow \quad \widehat{u}_{\lambda} \neq 0 .
\end{aligned}
$$

Also from (14) and (16) it follows that

$$
\left\|\widehat{u}_{\lambda}\right\|<\lambda^{\sigma} .
$$

Therefore $\widehat{u}_{\lambda} \in B_{\lambda} \backslash\{0\}$. On account of (15) we have

$$
\begin{aligned}
\widehat{u}_{\lambda} & \in K_{I_{\lambda}}, \\
& \Rightarrow \widehat{u}_{\lambda} \text { is a nontrivial solution of }(11), \lambda \in(0, \widehat{\lambda}) .
\end{aligned}
$$

From (17) we see that $\left\|u_{\hat{\lambda}}\right\| \rightarrow 0^{+}$as $\lambda \rightarrow 0^{+}$.

\section{References}

[1] A. Adams and J.F. Fournier, Sobolev Spaces, 2nd edn, Academic Press, 2003.

[2] C.O. Alves, A.R. de Holanda and J.A. Santos, Existence of positive solutions for a class of semipositone quasilinear problems through Orlicz-Sobolev space, Proc. Amer. Math. Soc. 147(1) (2019), 285-299. doi:10.1090/proc/14212.

[3] A. Bahrouni, V.D. Rădulescu and D.D. Repovš, Double phase transonic flow problems with variable growth: Nonlinear patterns and stationary waves, Nonlinearity 32(7) (2019), 2481-2495. doi:10.1088/1361-6544/ab0b03.

[4] D. Breit, Existence Theory for Generalized Newtonian Fluids, Mathematics in Science and Engineering., Elsevier/Academic Press, London, 2017. 
[5] M. Cencelj, V.D. Rădulescu and D.D. Repovš, Double phase problems with variable growth, Nonlinear Anal. 177 (2018), 270-287. doi:10.1016/j.na.2018.03.016.

[6] T.K. Donaldson and N.S. Trudinger, Orlicz-Sobolev spaces and imbedding theorems, J. Funct. Anal. 8 (1971), 52-75. doi:10.1016/0022-1236(71)90018-8.

[7] N. Fukagai, M. Ito and K. Narukawa, Positive solutions of quasilinear elliptic equations with critical Orlicz-Sobolev nonlinearity on $\mathbb{R}^{N}$, Funkcial. Ekvac. 49(2) (2006), 235-267. doi:10.1619/fesi.49.235.

[8] N. Fukagai and K. Narukawa, On the existence of multiple positive solutions of quasilinear elliptic eigenvalue problems, Ann. Mat. Pura Appl. (4) 186(3) (2007), 539-564. doi:10.1007/s10231-006-0018-x.

[9] J.P. Gossez, Orlicz-Sobolev Spaces and Nonlinear Elliptic Boundary Value Problems, Nonlinear Analysis, Function Spaces and Applications, BSB B.G. Teubner Verlagsgesellschaft, Leipzig, 1979, pp. 59-94, http://eudml.org/doc/220389.

[10] Y. Komiya and R. Kajikiya, Existence of infinitely many solutions for the $(p, q)$-Laplace equation, Nonlinear Differ. Equ. Appl. 23(4) (2016), 49.

[11] D. Motreanu, V.V. Motreanu and N.S. Papageorgiou, Topological and Variational Methods with Applications to Nonlinear Boundary Value Problems, Springer, New York, 2014.

[12] N.S. Papageorgiou, V.D. Rădulescu and D.D. Repovš, Double-phase problems with reaction of arbitrary growth, Z. Angew. Math. Phys. 69(4) (2018), 108.

[13] N.S. Papageorgiou, V.D. Rădulescu and D.D. Repovš, Double-phase problems and a discontinuity property of the spectrum, Proc. Amer. Math. Soc. 147(7) (2019), 2899-2910. doi:10.1090/proc/14466.

[14] N.S. Papageorgiou, V.D. Rădulescu and D.D. Repovš, Nonlinear Analysis - Theory and Methods, Springer Monographs in Mathematics, Springer, Cham, 2019.

[15] N.S. Papageorgiou, V.D. Rădulescu and D.D. Repovš, Ground state and nodal solutions for a class of double phase problems, Z. Angew. Math. Phys. 71(1) (2020), 15.

[16] N.S. Papageorgiou and C. Vetro, Superlinear $(p(z), q(z))$-equations, Complex Var. Elliptic Equ. 64(1) (2019), 8-25. doi:10.1080/17476933.2017.1409743.

[17] N.S. Papageorgiou, C. Vetro and F. Vetro, Solutions for parametric double phase Robin problems, Asymptot. Anal. (2020). doi:10.3233/ASY-201598.

[18] M.A. Ragusa and A. Tachikawa, Regularity for minimizers for functionals of double phase with variable exponents, $A d v$. Nonlinear Anal. 9(1) (2020), 710-728. doi:10.1515/anona-2020-0022.

[19] M.M. Rao and Z.D. Ren, Theory of Orlicz Spaces, Marcel Dekker, New York, 1991.

[20] Z. Tan and F. Fang, Orlicz-Sobolev versus Hölder local minimizer and multiplicity results for quasilinear elliptic equations, J. Math. Anal. Appl. 402(1) (2013), 348-370. doi:10.1016/j.jmaa.2013.01.029.

[21] C. Vetro, Semilinear Robin problems driven by the Laplacian plus an indefinte potential, Complex Var. Elliptic Equ. 65(4) (2020), 573-587. doi:10.1080/17476933.2019.1597066.

[22] F. Vetro, Infinitely many solutions for mixed Dirichlet-Neumann problems driven by the $(p, q)$-Laplace operator, Filomat 33(14) (2019), 4603-4611. doi:10.2298/FIL1914603V.

[23] Q. Zhang and V.D. Rădulescu, Double phase anisotropic variational problems and combined effects of reaction and absorption terms, J. Math. Pures Appl. 118 (2018), 159-203. doi:10.1016/j.matpur.2018.06.015. 